\title{
Telomerase catalytic subunit in laryngeal carcinogenesis-an immunohistochemical study
}

\author{
Boštjan Luzar ${ }^{1}$, Mario Poljak ${ }^{2}$ and Nina Gale ${ }^{1}$ \\ ${ }^{1}$ Institute of Pathology, Medical Faculty University of Ljubljana, Ljubljana, Slovenia and ${ }^{2}$ Institute of \\ Microbiology and Immunology, Medical Faculty University of Ljubljana, Ljubljana, Slovenia
}

\begin{abstract}
We recently demonstrated that (1) telomerase catalytic subunit messenger RNA (mRNA) relative quantities increase progressively with the degree of laryngeal epithelial abnormalities and that (2) telomerase catalytic subunit gene re-expression represents an early event in laryngeal carcinogenesis. The aim of the study was to determine whether telomerase catalytic protein immunohistochemisty reflects telomerase catalytic subunit gene expression in different grades of laryngeal epithelial abnormalities and squamous cell carcinomas of the larynx. Telomerase catalytic protein was analysed immunohistochemically in 106 laryngeal epithelial tissue samples: 10 normal epithelia, 15 squamous cell hyperplasias, 14 basal/parabasal cell hyperplasias, 10 atypical hyperplasias, eight intraepithelial carcinomas and 49 squamous cell carcinomas. At least 200 nuclei of each lesion were quantified per slide and the number of positive signals per nucleus was expressed as a telomerase catalytic protein index. The mean telomerase catalytic protein index increased progressively with the degree of laryngeal epithelial abnormalities: from 0.17 in normal epithelia, 0.44 in squamous cell hyperplasia, 0.54 in basal/parabasal cell hyperplasia, 0.91 in atypical hyperplasia, 1.05 in intraepithelial carcinoma to 0.96 in squamous cell carcinomas. Statistical analysis revealed three different groups of laryngeal epithelial changes according to the number of telomerase catalytic protein signals per nucleus: (1) normal epithelium, (2) regenerative epithelium (squamous cell hyperplasia, basal/parabasal cell hyperplasia), and (3) atypical hyperplasia, intraepithelial carcinoma and squamous cell carcinoma $(P<0.0033)$. Telomerase catalytic protein immunohistochemistry parallels well with telomerase catalytic subunit mRNA relative quantities in laryngeal carcinogenesis. In normal and regenerative laryngeal epithelia, telomerase catalytic protein is present in occasional basal/parabasal nuclei, becomes undetectable with maturation or differentiation of epithelial cells, and reflects the regenerative capacity of squamous epithelium. Nevertheless, several telomerase catalytic protein signals in the majority of nuclei in precancerous lesions, intraepithelial carcinomas and squamous cell carcinomas, are consistent with telomerase catalytic subunit gene re-expression, an early event in laryngeal carcinogenesis.

Modern Pathology (2005) 18, 406-411, advance online publication, 17 September 2004; doi:10.1038/modpathol.3800275
\end{abstract}

Keywords: carcinogenesis; hTERT protein; larynx; Ljubljana classification; squamous cell carcinoma; telomerase; telomerase catalytic subunit

Telomerase is a multisubunit enzyme complex functioning as a specialised reverse transcriptase that synthesises telomeric repeats to the $3^{\prime}$ ends of human chromosomes. ${ }^{1}$ The principal components of telomerase have been identified as the RNA template (hTR), telomerase associated protein (TEP1) and human telomerase reverse transcriptase (hTERT). ${ }^{2-5}$ While hTR and TEP1 are expressed

Correspondence: Assistant Professor B Luzar, MD, PhD, Institute of Pathology, Medical Faculty University of Ljubljana, Korytkova 2, 1000 Ljubljana, Slovenia.

E-mail: bostjan.luzar@mf.uni-lj.si

Received 11 June 2004; revised and accepted 5 August 2004; published online 17 September 2004 ubiquitously, hTERT protein expression has been found to be highly regulated and generally correlates with telomerase activity. ${ }^{6-8}$

The 40-kb single copy hTERT gene, mapping to chromosome 5p15.33, codes for a 127-kDa protein of 1132 amino acids contained in 16 exons. ${ }^{5}$ Several splice variants of the $h$ TERT gene transcript have been described. ${ }^{9,10}$ However, it is generally accepted that only the full-length transcript of the $h T E R T$ gene can function as the catalytic subunit of telomerase, since it contains the whole core catalytic domain and the specialised carboxy-terminus of this transcriptase. ${ }^{11}$

We have recently demonstrated that telomerase catalytic subunit (hTERT) mRNA relative quantities 
increase progressively with the degree of laryngeal epithelial abnormalities by using RT-PCR, and hypothesised that hTERT gene re-expression represents an early event in the multistep process of laryngeal carcinogenesis. ${ }^{12,13}$ The aim of the present study was, therefore, to analyse whether hTERT protein immunohistochemistry parallels hTERT mRNA relative quantities, for example, reflects hTERT gene expression, in different morphological grades of laryngeal epithelial abnormalities and squamous cell carcinoma of the larynx.

\section{Materials and methods}

\section{Patients and Tissues}

Biopsy tissue samples of laryngeal squamous cell carcinomas from 49 patients, obtained in the period from 1998 to 2003, were retrieved from the archive of the Institute of Pathology, Medical Faculty, Ljubljana. In the vicinity of each laryngeal squamous cell carcinoma, the laryngeal epithelium showed various degrees of epithelial abnormalities, and was evaluated separately. Accordingly, 49 samples of laryngeal squamous cell carcinomas and 48 laryngeal epithelial samples with different grades of epithelial abnormalities were obtained. All biopsies were taken before patients received any treatment.

Normal laryngeal epithelial tissue samples were obtained at autopsies from 10 patients, who died due to unrelated causes.

Laryngeal tissue blocks were fixed in formalin and embedded in paraffin. Standard haematoxylin and eosin-stained sections were used to establish the diagnosis and to grade the tumours into well (grade I), moderately (grade II) and poorly (grade III) differentiated laryngeal squamous cell carcinomas according to the WHO classification. ${ }^{14}$ In addition, laryngeal epithelial abnormalities were classified according to the Ljubljana classification of laryngeal epithelial hyperplastic lesions into four groups: (1) squamous cell hyperplasia (simple hyperplasia), (2) basal/parabasal cell hyperplasia (abnormal hyperplasia), (3) atypical hyperplasia, and (4) intraepithelial carcinoma. ${ }^{15,16}$

\section{Immunohistochemical Detection of hTERT Protein}

The presence of hTERT protein was investigated by immunohistochemistry. Serial sections of $4 \mu \mathrm{m}$ thickness were deparaffinized, rehydrated and washed in PBS for $15 \mathrm{~min}$. Antigen retrieval and staining with monoclonal antibody NCL-hTERT (Novocastra, UK), diluted 1:20, was performed in an automatic immunostainer Discovery (Ventana, USA). After washing, sections were treated with biotinylated secondary antibody for $30 \mathrm{~min}$ followed by incubation with peroxidase-conjugated streptavidin for $30 \mathrm{~min}$. Visualisation of the immunoreac- tion was conducted with $3,3^{\prime}$-diaminobenzidine (Ventana detection kit, Ventana, USA) for $5 \mathrm{~min}$. Finally, sections were counterstained with haematoxylin. Sections treated without primary antibodies served as negative controls. Normal human tonsil and testicular tissue were used as positive controls for hTERT protein.

\section{Quantification of hTERT Protein}

If present, telomerase is associated with telomeres at the very ends of human chromosomes. A positive signal for hTERT protein was therefore identified as a clear $\operatorname{dot}(\mathrm{s})$ within the nuclei of laryngeal epithelial cells. Theoretically, depending on the quantity of hTERT protein, isolated small $\operatorname{dot}(\mathrm{s})$ or several large $\operatorname{dot}(\mathrm{s})$ could be observed within the nuclei of laryngeal epithelial cells. At least 200 nuclei of the corresponding epithelial lesion were quantified per slide and the number of positive signals per nucleus was expressed as an hTERT protein index.

In the corresponding epithelial lesion, nuclei were counted throughout the epithelial thickness.

\section{Statistical Analysis}

Statistical analysis was performed by a nonparametrical Mann-Whitney U-test (nonparametrical Wilcoxon test) with Bonferroni correction using SPSS 11.0 for Windows, $P$ values lower than 0.0033 were regarded as statistically significant.

\section{Results}

The presence of hTERT protein was analysed in a total of 106 laryngeal tissue samples: 10 normal epithelia, 15 squamous cell hyperplasias (simple hyperplasias), $14 \mathrm{basal} /$ parabasal hyperplasias (abnormal hyperplasias), 10 atypical hyperplasias, eight intraepithelial carcinomas and 49 squamous cell carcinomas (14 grade I, 20 grade II and 15 grade III).

In normal laryngeal epithelium, hTERT protein immunoreactivity was usually negative, or was occasionally present within the nuclei of the basal/ parabasal epithelial layer, but not in the spinous layer (Figure 1). In squamous and basal/parabasal cell hyperplasia, the nuclei of basal/parabasal cells showed occasional hTERT protein immunoreactivity (Figure 2).

In contrast, atypical hyperplasia was characterised by diffuse hTERT protein-positive nuclei usually extending up to two-thirds of the epithelial thickness (Figure 3), sometimes throughout. Several dots were observed within the same nuclei. Similarly, several hTERT protein positive signals that were also of different sizes and shapes were found within the same nucleus in intraepithelial 


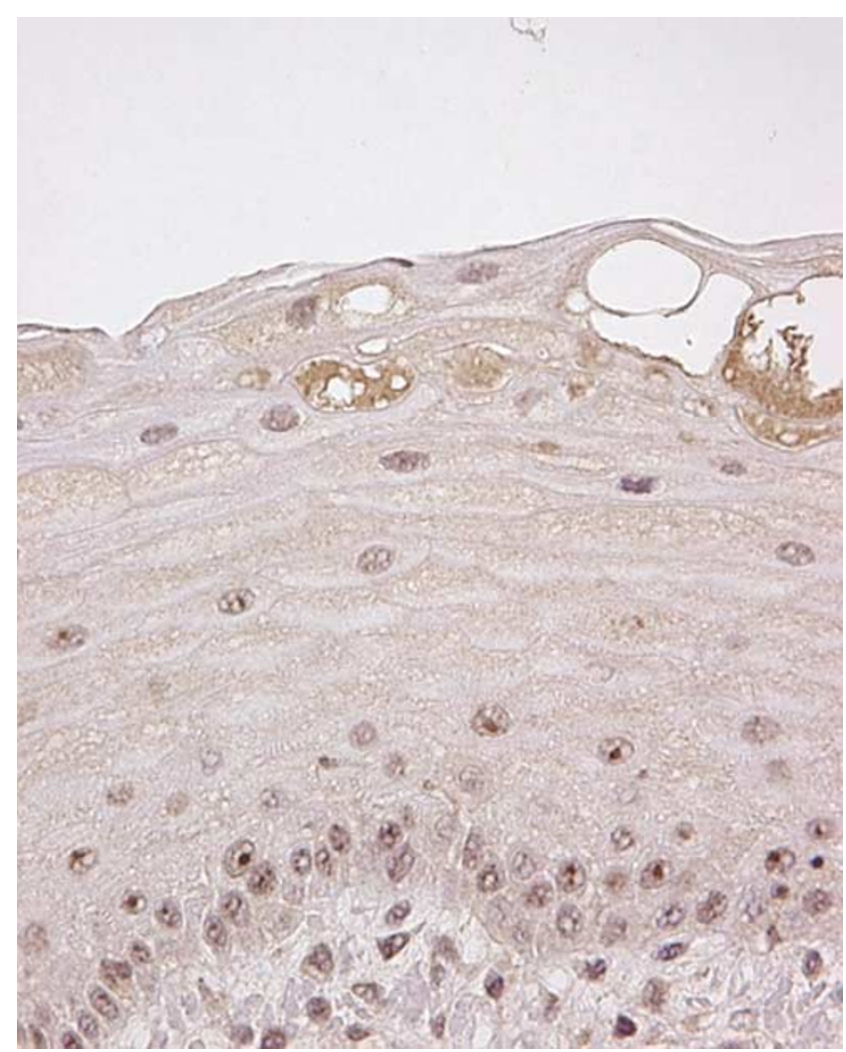

Figure 1 Normal laryngeal epithelium. Isolated hTERT protein signals are seen in the nuclei of basal cells.



Figure 2 Reactive hyperplastic laryngeal lesion (basal/parabasal cell hyperplasia). Occasional hTERT positive nuclear signals are seen in basal/parabasal cells. Note the absence of hTERT positivity in the upper epithelial layers.

carcinomas and laryngeal squamous cell carcinomas (Figures 4 and 5). The distribution of hTERT protein positive nuclei was uneven within laryngeal squamous cell carcinomas.

The mean number of hTERT protein signals per nucleus increased progressively with the degree of

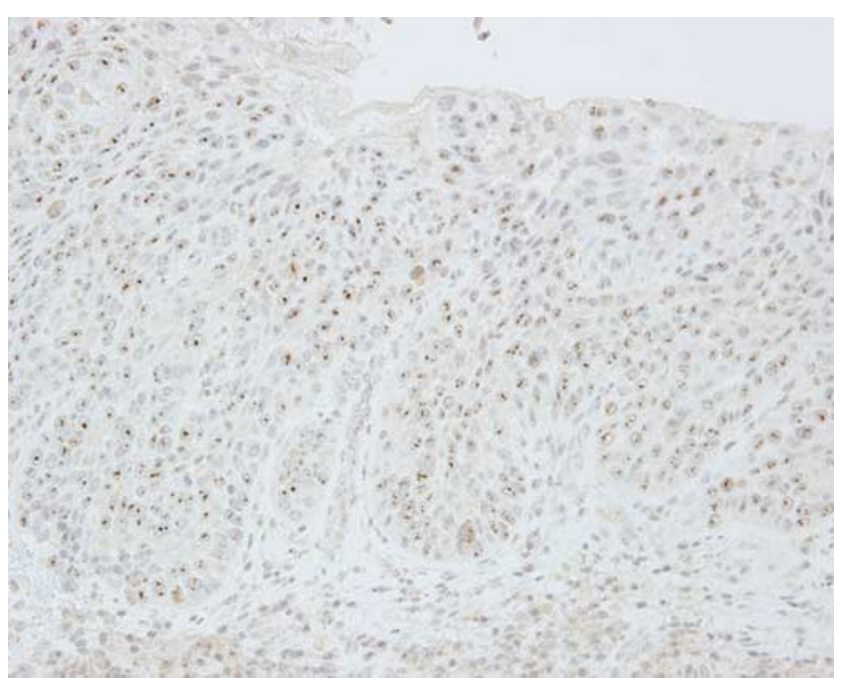

Figure 3 Atypical hyperplasia (precancerous lesion). Several hTERT protein signals are present within individual nuclei throughout the epithelial thickness.

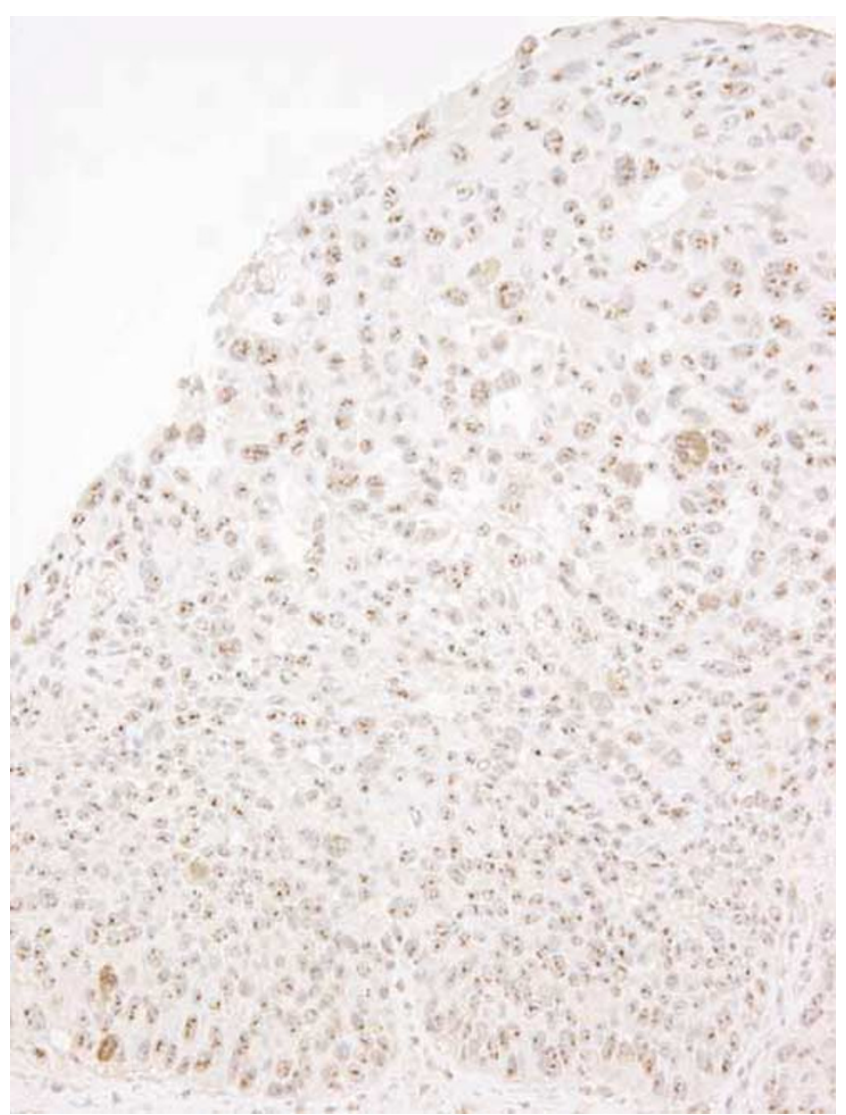

Figure 4 Carcinoma in situ. Several hTERT protein signals are observed within individual nuclei throughout the epithelial thickness.

laryngeal epithelial abnormalities: from 0.17 in normal epithelia, 0.44 in squamous cell hyperplasia, $0.54 \mathrm{in} \mathrm{basal/parabasal} \mathrm{cell} \mathrm{hyperplasia,} 0.91$ in atypical hyperplasia, 1.05 in intraepithelial carcinoma to 0.96 in laryngeal squamous cell carcinomas 


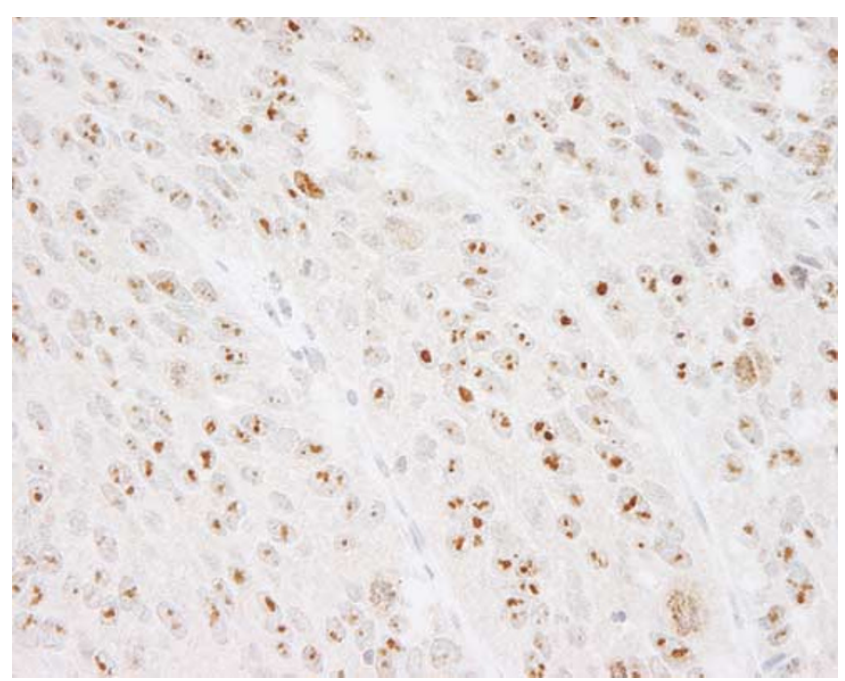

Figure 5 Squamous cell carcinoma. hTERT protein immunoreactivity is present in the majority of neoplastic nuclei. Note several small and large dots within the same nuclei.

Table 1 hTERT indices in normal laryngeal epithelium, different grades of laryngeal epithelial abnormalities and invasive squamous cell carcinomas of the larynx

\begin{tabular}{|c|c|c|c|c|}
\hline \multirow[t]{2}{*}{ Laryngeal tissue } & \multirow{2}{*}{$\begin{array}{l}\text { No. of } \\
\text { cases }\end{array}$} & \multicolumn{3}{|c|}{ hTERT index } \\
\hline & & Mean & Range & $\begin{array}{l}\text { Standard } \\
\text { deviation }\end{array}$ \\
\hline Normal epithelium & 10 & 0.17 & $0.03-0.44$ & 0.14 \\
\hline $\begin{array}{l}\text { Squamous cell } \\
\text { hyperplasia }\end{array}$ & 15 & 0.44 & $0.21-0.63$ & 0.12 \\
\hline $\begin{array}{l}\text { Basal and parabasal } \\
\text { cell hyperplasia }\end{array}$ & 14 & 0.54 & $0.34-0.72$ & 0.10 \\
\hline Atypical hyperplasia & 10 & 0.91 & $0.74-1.08$ & 0.12 \\
\hline Carcinoma in situ & 8 & 1.05 & $0.79-1.65$ & 0.29 \\
\hline $\begin{array}{l}\text { Squamous cell } \\
\text { carcinoma }\end{array}$ & 49 & 0.96 & $0-1.99$ & 0.35 \\
\hline
\end{tabular}

(1.00 in grade I, 0.90 in grade II and 0.99 in grade III (Table 1).

Statistical analysis revealed that three different groups of laryngeal epithelial changes can be separated according to the number of hTERT protein signals per nucleus: (1) normal epithelium, (2) regenerative epithelium (squamous cell hyperplasia, basal/parabasal cell hyperplasia), and (3) atypical hyperplasia, intraepithelial carcinoma and LC $(P<0.001)$. Furthermore, no significant differences existed in the number of hTERT protein signals per nucleus between well, moderately and poorly differentiated laryngeal squamous cell carcinomas $(P>0.05)$.

\section{Discussion}

Normal human somatic cells, with the exception of germ cells and stem cells of renewable tissues, generally lack telomerase activity. ${ }^{17}$ In contrast, telomerase reactivation has been observed in the majority of human malignancies. ${ }^{17}$ While telomerase is a multisubunit enzyme complex, it appears that up- and downregulation of its catalytic subunit is associated with telomerase activity, or its absence. ${ }^{5}$ It has been hypothesised that reactivation of telomerase is one of the most frequent events in human carcinogenesis, associated with cellular immortality, thus promoting the accumulation of genetic abnormalities and increasing genomic instability. ${ }^{5,18}$

Head and neck squamous cell carcinoma is the sixth most frequent cancer, accounting for $6 \%$ of all cancers worldwide. ${ }^{19}$ Laryngeal and hypopharyngeal squamous cell carcinomas form the largest subset of this group, usually occurring in older males. ${ }^{19}$ Despite improvements in the diagnosis and treatment, the mortality rate has remained relative stable over the last two decades, with an overall death risk as high as $40 \%$ in the subsequent $2-5$ years. ${ }^{20}$

From 6 to 10 independent genetic events within the single cell have been estimated to be necessary for squamous cell carcinoma in the head and neck region. ${ }^{21}$ They are believed to be morphologically expressed as different grades of laryngeal epithelial abnormalities. According to the Ljubljana classification of epithelial hyperplastic laryngeal lesions, they range from benign (squamous cell hyperplasia and basal/parabasal cell hyperplasia), potentially malignant (atypical hyperplasia or precancerous lesion) to intraepithelial carcinoma (carcinoma in situ). ${ }^{15,16}$ While the risk of progression to squamous cell carcinoma in the subsequent 10 years for squamous cell hyperplasia and basal/parabasal cell hyperplasia is low (1.0 and $0.9 \%$, respectively), it rises sharply for atypical hyperplasia $(10 \%)$, and may well be as high as $90 \%$ for intraepithelial carcinoma. ${ }^{15,16,22}$

We have previously shown by RT-PCR that telomerase reactivation, measured indirectly by the levels of hTERT messenger RNA (mRNA), is an early event in laryngeal carcinogenesis, detectable already at the stage of precancerous lesions. ${ }^{12}$ Although the RT-PCR is sensitive for detecting even small quantities of hTERT mRNA, its main drawback is that the tissue morphology is destroyed, thereby preventing the detection of particular cells with telomerase activity. It is well known that, in addition to neoplastic cells, activated lymphocytes do possess low levels of telomerase activity and may influence RT-PCR results. ${ }^{23}$ To the best of our knowledge, the present study is the first to analyse systematically the presence of hTERT protein in different morphological grades of laryngeal carcinogenesis, including laryngeal squamous cell carcinomas, by immunohistochemistry.

\section{hTERT Protein in Normal Laryngeal Epithelium}

Normal laryngeal epithelium is characterised morphologically by one or two rows of basal cells and up to five rows of prickle cell layers. ${ }^{22}$ The basal epithelial layer has been known to represent less 
differentiated cells than the prickle cell layer, where cells become terminally differentiated and gradually lose their dividing potential as they mature towards the surface of the epithelium. ${ }^{22}$ In the present study, hTERT protein was usually absent in normal laryngeal epithelia, but if present, the hTERT protein was located in the nuclei of occasional basal cells. Basal cells are committed descendents of epithelial stem cells, and may possess low levels of telomerase activity for regenerative purposes. ${ }^{24} \mathrm{In}$ agreement with the low number of hTERT protein signals per nucleus in normal laryngeal epithelium, as shown by immunohistochemistry, we previously detected low relative quantities of hTERT mRNA in just $7 \%$ of normal laryngeal epithelia. ${ }^{12}$

\section{hTERT Protein in Regenerative (Squamous and Basal/ Parabasal Hyperplasia) Laryngeal Epithelium}

Significantly higher hTERT protein indices were calculated in squamous and basal/parabasal cell hyperplasia of the laryngeal epithelium than in normal laryngeal epithelia. Nevertheless, the number of dots per nucleus in these two reactively hyperplastic laryngeal epithelia was similar. Interestingly, although the relative quantities of hTERT mRNA detected by RT-PCR in squamous and basal/ parabasal cell hyperplasia were higher than in normal laryngeal epithelia, the relative quantities were still low and the differences were not statistically significant. ${ }^{12}$ Morphologically, only nuclei of basal and parabasal cells showed hTERT protein immunoreactivity in squamous and basal/parabasal cell hyperplasia, mostly as one single small dot, while the nuclei in the spinous (prickle cell) layer were negative. These data suggest that hTERT protein becomes undetectable with maturation or differentiation of laryngeal epithelial cells, reflecting inhibition of hTERT gene trascription and the disappearance of telomerase activity. When studying proliferative activity in laryngeal hyperplastic lesions, Zidar et $a l^{25}$ discovered only occasional proliferating cells in the basal layer in squamous cell hyperplasia, while basal/parabasal hyperplasia was characterised by proliferating cells occupying the lower third of the epithelial thickness. ${ }^{25}$ These data additionally support our hypothesis that low hTERT indices (eg low number of hTERT protein signals per nucleus) most likely correspond to low levels of telomerase activity providing regenerative capacity to the squamous epithelium, and do not reflect reexpression of the hTERT gene as a part of the multistep process of laryngeal carcinogenesis.

\section{hTERT Protein in Atypical Hyperplasia, Intraepithelial Carcinoma and Squamous Cell Carcinoma of the Larynx}

Atypical hyperplasia has a central position in the Ljubljana classification of laryngeal epithelial hyperplastic lesions and has been regarded as a potentially malignant lesion or 'precancerous lesion', since a malignant transformation up to $10 \%$ was observed in patients with atypical hyperplasia during a more than 10-year follow-up period. ${ }^{15,16,22}$ Atypical hyperplasia is characterised morphologically by pronounced cytological abnormalities, while epithelial stratification remains preserved: nuclei of many epithelial cells show the changes of 'atypia', such as nuclear enlargement with irregular contours, hyperchromasia, increased nuclear/cytoplasmic ratio and an increased number of nucleoli. ${ }^{15}$ Mitoses are increased and are usually found in the lower two-thirds of the epithelium. ${ }^{15}$ Intraepithelial carcinoma, on the other hand, already has all the morphological features of malignancy but lacks basement membrane penetration and invasive growth. While the number of hTERT protein signals per nucleus in atypical hyperplasia, intraepithelial carcinoma and laryngeal squamous cell carcinoma was significantly higher than in normal and regenerative laryngeal epithelium, the differences between atypical hyperplasia, intraepithelial carcinoma and laryngeal squamous cell carcinoma were not statistically significant. In agreement, the pattern of hTERT protein immunoreactivity was similar in atypical hyperplasia, intraepithelial carcinoma and laryngeal squamous cell carcinoma. Several hTERT protein positive signals in the form of small and/or large oval to rounded dots were found within the nuclei throughout the epithelial thickness and/or laryngeal squamous cell carcinoma, although the distribution of immunoreactivity was occasionally uneven within the particular lesion. The hTERT protein positive cells were of dysplastic morphology. These data support the results of our previous study, in which we detected significantly higher relative quantities of hTERT mRNA in atypical hyperplasia, intraepithelial carcinoma and invasive laryngeal squamous cell carcinoma than in reactive hyperplastic laryngeal epithelia. ${ }^{12}$ We believe that significantly higher hTERT protein signals per nucleus in precancerous lesions (atypical hyperplasia), intraepithelial and laryngeal squamous cell carcinomas are the consequence of $h T E R T$ gene re-expression as a part of the multistep process of laryngeal carcinogenesis. Since no statistically significant differences exist in the numbers of hTERT protein signals per nucleus between atypical hyperplasia, intraepithelial and invasive squamous cell carcinoma of the larynx, other genetic abnormalities than telomerase reactivation must be responsible for their development and/or progression.

In conclusion, hTERT protein immunohistochemistry parallels well with hTERT mRNA relative quantities in laryngeal carcinogenesis. In normal and regenerative laryngeal epithelia, hTERT protein is present in occasional basal/parabasal nuclei, becomes undetectable with maturation or differentiation of laryngeal epithelial cells, and reflects the 
regenerative capacity of laryngeal squamous epithelium. Nevertheless, the presence of one or, even more commonly, several hTERT signals in the majority of nuclei in precancerous lesions (atypical hyperplasias), intraepithelial carcinomas and laryngeal squamous cell carcinomas, is consistent with hTERT gene re-expression, an early event in laryngeal carcinogenesis.

\section{References}

1 Shippen-Lentz D, Blackburn EH. Functional evidence for RNA template in telomerase. Science 1990;247: 546-552.

2 Feng J, Funk WD, Wang SS, et al. The RNA component of human telomerase. Science 1995;269:1236-1241.

3 Nakamura TM, Morin GB, Chapman KB, et al. Telomerase catalytic subunit homologs from fission yeast and human. Science 1997;277:955-959.

4 Saito T, Matsuda Y, Suzuki T, et al. Comparative gene mapping of the human and mouse TEP1 genes, which encode one protein component of telomerases. Genomics 1997;46:46-50.

5 Meyerson M, Counter CM, Eaton EN, et al. hEST2, the putative human telomerase catalytic subunit gene, is up-regulated in tumor cells and during immortalization. Cell 1997;90:85-95.

6 Ito H, Kyo S, Kanaya T, et al. Expression of human telomerase subunits and correlation with telomerase activity in urothelial cancer. Clin Cancer Res 1998; 4:1603-1608.

7 Counter CM, Meyerson M, Eaton EN, et al. Telomerase activity is restored in human cells by ectopic expression of hTERT (hEST2), the catalytic subunit of telomerase. Oncogene 1998;16:1217-1222.

8 Koyanagi Y, Kobayashi D, Yajima T, et al. Telomerase activity is down regulated via decreases in hTERT mRNA but not TEP1 mRNA or hTERC during the differentiation of leukemic cells. Anticancer Res 2000;20:773-778.

9 Kilian A, Bowtell DD, Abud HE, et al. Isolation of a candidate human telomerase catalytic subunit gene, which reveals complex splicing patterns in different cell types. Hum Mol Genet 1997;6:2011-2019.

10 Ulaner GA, Hu JF, Vu TH, et al. Tissue-specific alternate splicing of human telomerase reverse transcriptase (hTERT) influences telomere lengths during human development. Int J Cancer 2001;91:644-649.

11 Kotoula V, Hytiroglou P, Pyrpasopoulou A, et al. Expression of human telomerase reverse transcriptase in regenerative and precancerous lesions of cirrhotic livers. Liver 2002;22:57-69.
12 Luzar B, Poljak M, Marin IJ, et al. Telomerase reactivation is an early event in laryngeal carcinogenesis. Mod Pathol 2003;16:841-848.

13 Luzar B, Poljak M, Marin IJ, et al. Quantitative measurement of telomerase catalytic subunit (hTERT) mRNA in laryngeal squamous cell carcinomas. Anticancer Res 2001;21:4011-4015.

14 Shanmugaratnam K, Sobin LH. Histological typing of tumours of the upper respiratory tract and ear. World Health Organization-International Histological Classification of Tumours, 2nd edn. Springer Verlag: Berlin, 1991.

15 Hellquist H, Cardesa A, Gale N, et al. Criteria for grading in the Ljubljana classification of epithelial hyperplastic laryngeal lesions. A study by members of the Working Group on Epithelial Hyperplastic Laryngeal Lesions of the European Society of Pathology. Histopathology 1999;34:226-233.

16 Gale N, Kambič V, Michaels L, et al. The Ljubljana classification: a practical strategy for the diagnosis of laryngeal precancerous lesions. Adv Anat Pathol 2000;7:240-251.

17 Dhaene K, Van Marck E, Parwaresch R. Telomeres, telomerase and cancer: an up-date. Virchows Arch 2000;437:1-16.

18 Takakura M, Kyo S, Kanaya T, et al. Cloning of human telomerase catalytic subunit (hTERT) gene promoter and identification of proximal core promoter sequences essential for transcriptional activation in immortalized and cancer cells. Cancer Res 1999;59: 551-557.

19 Parkin DM, Pisani P, Ferlay J. Estimates of the worldwide incidence of eighteen major cancers in 1985. Int J Cancer 1993;54:594-606.

20 van der Sanden GA, Coebergh JW, Schouten LJ, et al. Cancer incidence in The Netherlands in 1989 and 1990: first results of the nationwide Netherlands cancer registry. Coordinating Committee for Regional Cancer Registries. Eur J Cancer 1995;31A:1822-1829.

21 Renan MJ. How many mutations are required for tumorigenesis? Implications from human cancer data. Mol Carcinogen 1993;7:139-146.

22 Kambič V, Gale N. Epithelial hyperplastic lesions of the larynx. Amsterdam: Elsevier, 1995.

23 Liu K, Schoonmaker MM, Levine BL, et al. Constitutive and regulated expression of telomerase reverse transcriptase (hTERT) in human lymphocytes. Proc Natl Acad Sci USA 1999;96:5147-5152.

24 Fujimoto R, Kamata N, Yokoyama K, et al. Expression of telomerase components in oral keratinocytes and squamous cell carcinomas. Oral Oncol 2001;37:132-140.

25 Zidar N, Gale N, Cör A, et al. Expression of Ki-67 antigen and proliferative cell nuclear antigen in benign and malignant epithelial lesions of the larynx. J Laryngol Otol. 1996;110:440-445. 